\title{
The Spherical Hierarchical Model
}

\author{
J. B. McGuire \\ Department of Physics, Florida Atlantic University, Boca Raton, Florida, USA
}

Received March 8, 1973

\begin{abstract}
The spherical version of Dyson's hierarchical model is analyzed. A particular case which is designed to simulate the long-range Ising problem is dealt with in detail. A phase transition is found with critical temperature

$$
\beta_{c}=\frac{1}{2}\left(2^{\alpha}-2\right)\left(4-2^{\alpha}\right)^{-1}
$$

where $n^{\text {th }}$ neighbor spins interact with a strength of $n^{-\alpha}$. Critical exponents are calculated for this particular case and are found to be identical with the critical exponents of the long-range spherical Ising model.
\end{abstract}

\section{Introduction}

The hierarchical model suggested by Dyson [1] is defined as follows: "There are $2^{p}$ spins $\mu_{j}= \pm 1$, labelled by the index $j=1,2 \ldots 2^{N}$. For each pair of integers $p=0,1,2 \ldots N ; r=1,2 \ldots 2^{N-p}$ we consider the spin sum

$$
S_{p, r}=\sum_{j} \mu_{j}, \quad(r-1) 2^{p}+1 \leqq j \leqq r 2^{p} .
$$

This is the sum of the $r^{\text {th }}$ block of $2^{p}$ consecutive spins. The hierarchical character of these sums is expressed by the relation

$$
S_{p, r}=S_{p-1,2 r-1}+S_{p-1,2 r}, \quad p=1,2 \ldots N .
$$

We assume the interaction energy in the model to be

$$
H_{N}=-\sum_{p=1}^{N} 2^{-2 p} b_{p} \sum_{r=1}^{2^{N-p}}\left(S_{p, r}\right)^{2}
$$

where $b_{1} \ldots b_{N}$ are non-negative coefficients. The statistical properties of the model are completely defined given the above and a temperature $T=(k \beta)^{-1}$."

Dyson is able to show that much useful information about the Ising model with long range interactions can be obtained by analyzing the hierarchical model and connecting the hierarchical model to the Ising model through the inequalities of Griffiths [2]. Dyson was not able, nor are we able, to solve the hierarchical model directly. In this paper we 
shall be more modest in our goal and restrict our considerations to the spherical version of the hierarchical model, that is the version of this problem which bears the same relation to this problem that the spherical model [3] bears to the Ising problem.

We shall find that there are many interesting features to this simplified problem. Among these features are the existence of a phase transition whose critical temperature we shall compare with the bounds established by Dyson. We shall further compare our results with the detailed analysis of the spherical Ising model with long range interactions carried out by Joyce [3].

\section{The Spherical Hierarchical Model}

The hierarchical partition function may be thought of as the sum of $\exp \left(-\beta H_{N}\right)$ over the allowed values of $H_{N}$. These allowed values of $H_{N}$ are functions of the random variables $\mu_{j}= \pm 1$. In $\mu_{j}$ space therefore, the partition function is a discret sum over the verticies of a $2^{N}$ dimensional hyper-cube. The spherical hierarchical model replaces the sum over the verticies of this cube by an integral over the sphere which circumscribes it.

Thus the partition function for the spherical hierarchical model is the restricted integral

$$
Q_{N}(\beta)=\int \ldots \int d \mu_{1} \ldots d \mu_{2^{N}} \exp \left(-\beta H_{N}\right),
$$

where the $\mu_{j}$ are constrained by

$$
\mu_{1}^{2}+\mu_{2}^{2}+\cdots+\mu_{2^{N}}^{2}=2^{N} .
$$

This integral may be calculated most easily by this introduction of an undetermined multiplier, or, equivalently, by the use of the grand canonical ensemble. We write

$$
Q_{N}(\mu, \beta)=\int_{-\infty}^{\infty} \ldots \int_{-\infty}^{\infty} d \mu_{1} \ldots d \mu_{2^{N}} \exp \left(-\beta H_{N}-\mu \sum_{i=1}^{2^{N}} \mu_{i}^{2}\right)
$$

and the undetermined multiplier $\mu$, is determined through the auxiliary equation

$$
2^{N}=-\frac{\partial}{\partial \mu} \ln Q_{N}(\mu \beta) .
$$

The usual arguments hold, viz. in the limit of large $N$ Eq. (6) is essentially equivalent to Eq. (4).

The hierarchical Hamiltonian is a negative definite quadratic form in the $\mu_{j}$ because the Hamiltonian itself is quadratic in the $S_{p, r}$ and the recurrence relation (2) is linear.

The simplifying feature of the spherical constraint arises from the fact that since $H_{N}$ is a negative definite quadratic form in the random 
variables $\mu_{j}$ it may be put in diagonal form by an orthogonal transformation. This orthogonal transformation will leave the spherical constraint unaffected, and we may evaluate all of the integrals in (5) provided that the appropriate orthogonal transformation can be found.

When the orthogonal transformation is found the partition function will be

$$
Q_{N}(\mu, \beta)=\int_{-\infty}^{\infty} \ldots \int_{-\infty}^{\infty} d x_{1} \ldots d x_{2^{N}} \exp \left(\beta \sum_{k=1}^{2^{N}} h_{k} x_{k}^{2}-\mu \sum_{k=1}^{2^{N}} x_{k}^{2}\right)
$$

where the $h_{k}$ are the characteristic values, and the $x_{k}$ are the normal coordinates of $H_{N}$. Evaluation of the integrals in $Q_{N}$ is elementary and leads to a free energy, $\psi_{N}$, given by

$$
-\beta \psi_{N}=\ln Q_{N}(\mu, \beta)=\frac{-1}{2} \sum_{k=1}^{2^{N}} \ln \left(\mu-\beta h_{k}\right)+2^{N-1} \ln \pi
$$

where the relation between $\beta$ and $\mu$ is

$$
2^{N}=-\frac{\partial}{\partial \mu} \ln Q_{N}(\mu, \beta)=\frac{1}{2} \sum_{k=1}^{2 N}\left(\mu-\beta h_{k}\right)^{-1} .
$$

We shall find it convenient to modify Dyson's Hamiltonian slightly and let

with

$$
H_{N}=-\sum_{p=0}^{N} 2^{-2 p} b_{p} \sum_{r=1}^{2^{N-p}}\left(S_{p, r}\right)^{2}
$$

$$
S_{0, r}=\mu_{r} .
$$

We have added a "self energy" term of

$$
b_{0} \sum_{k=1}^{2 N} \mu_{k}^{2}=2^{N} b_{0}
$$

to the Hamiltonian. We recover Dyson's hamiltonian if we set $b_{0}=0$.

We now represent the Hamiltonian as

$$
H_{N}=U^{\dagger} A_{N} U,
$$

where $U$ is a column vector with the $\mu_{j}$ as components, and $A_{N}$ is a $2^{N} \times 2^{N}$ symmetric matrix with functions of the $b$ 's as elements.

The hierarchical nature of the interaction manifests itself in the $A_{N}$. We write down the first two $A$ 's:

$$
\begin{aligned}
& A_{0}=b_{0} \\
& A_{1}=\left[\begin{array}{lr}
b_{0}+2^{-2} b_{1} & 2^{-2} b_{1} \\
2^{-2} b_{1} & b_{0}+2^{-2} b_{1}
\end{array}\right] .
\end{aligned}
$$


If we let $C_{N}$ be a $2^{N} \times 2^{N}$ matrix with all elements equal to unity, then the recurrence relation which duplicates the Hamiltonian [3] is

$$
A_{N}= \begin{cases}A_{N-1}+2^{-2 N} b_{N} C_{N-1} & 2^{-2 N} b_{N} C_{N-1} \\ 2^{-2 N} b_{N} C_{N-1} & A_{N-1}+2^{-2 N} b_{N} C_{N-1} .\end{cases}
$$

It is easy to show that $A_{N}$ and $C_{N}$ commute if $A_{N-1}$ and $C_{N-1}$ commute, and thus all of the $A$ 's commute with the $C$ 's because $A_{0}$ and $C_{0}$ commute. Therefore, we may simultaneously diagonalize both $A$ and $C$.

Note that any eigenvector of $A_{N-1}$ and $C_{N-1}$ leads to two eigenvectors of $A_{N}$ and $C_{N}$. Suppose that

$$
\begin{aligned}
& A_{N-1} v=a_{N-1} v, \\
& C_{N-1} v=c_{N-1} v .
\end{aligned}
$$

From this we see that

and

$$
A_{N}\left(\begin{array}{l}
V \\
V
\end{array}\right)=\left(a_{N-1}+2^{1-2 N} b_{N} C_{N-1}\right)\left(\begin{array}{l}
V \\
V
\end{array}\right)
$$

$$
A_{N}\left(\begin{array}{c}
V \\
-V
\end{array}\right)=a_{N-1}\left(\begin{array}{c}
V \\
-V
\end{array}\right) \text {. }
$$

The matrix $A_{0}$ has one eigenvector, viz. 1 . From this we may construct the two eigenvectors for $A_{1}$. They are:

with eigenvalues

$$
V_{1}=2^{-1 / 2}\left(\begin{array}{l}
1 \\
1
\end{array}\right), \quad V_{2}=2^{-1 / 2}\left(\begin{array}{c}
1 \\
-1
\end{array}\right)
$$

$$
h_{1}=b_{0}+2^{-1} b_{1}, \quad h_{2}=b_{0} .
$$

From this pair of eigenvectors and eigenvalues we may construct the eigenvectors and eigenvalues of $A_{2}$ etc.

By continuing this process we find that $A_{N}$ has $2^{N}$ eigenvalues arranged as follows:

$$
\begin{array}{cc}
h_{0}=b_{0} & 2^{N-1} \text { fold degenerate } \\
h_{1}=b_{0}+2^{-1} b_{1} & 2^{N-2} \text { fold degenerate } \\
\vdots & \vdots \\
h_{k}=\sum_{p=0}^{k} 2^{-p} b_{p} & 2^{N-(k+1)} \text { fold degenerate } \\
\vdots & \\
h_{N}=\sum_{p=0}^{N} 2^{-p} b_{p} & \text { non-degenerate. }
\end{array}
$$


We could also compute all of the normal coordinates in terms of the $\mu_{j}$. This will turn out not to be necessary, for we shall only require the normal coordinate associated with the largest eigenvalue of $A_{N}$. We shall call this coordinate $X_{1}$, and in normalized form it is given by

$$
X_{1}=2^{-N} \sum_{j=1}^{2 N} \mu_{j}
$$

The partition function integrals are performed as in (7). The result is

$-\beta \psi_{N}=\ln Q_{N}$

$=-2^{N}\left[\frac{1}{4} \sum_{k=0}^{N} 2^{-k} \ln \left(\mu-\beta \sum_{p=0}^{k} 2^{-p} b_{p}\right)+\frac{1}{4} 2^{-N} \ln \left(\mu-\beta \sum_{p=0}^{N} 2^{-p} b_{p}\right)\right]$,

$2^{N}=2^{N}\left[\frac{1}{4} \sum_{k=0}^{N} 2^{-k}\left(\mu-\beta \sum_{p=0}^{k} 2^{-p} b_{p}\right)^{-1}+\frac{1}{4} 2^{-N}\left(\mu-\beta \sum_{p=0}^{N} 2^{-p} b_{p}\right)\right]$.

The second term on the right in each of these expressions comes from the largest eigenvalue $h_{N}$. This term will not have any appreciable effect in the limit of large $N$, so we suppress it. It is also convenient to define the variable $s$ such that

$$
\mu=\beta \sum_{p=0}^{\infty} 2^{-p} b_{p}(s+1) .
$$

With these two revisions our equations read:

where

$$
\begin{gathered}
2^{-N} \beta \psi_{N}=\frac{1}{4} \sum_{k=0}^{N} 2^{-k} \ln \beta A\left(s+A^{-1} \sum_{p=k+1}^{\infty} 2^{-p} b_{p}\right) \\
\beta A=\frac{1}{4} \sum_{k=0}^{N} 2^{-k}\left(s+A^{-1} \sum_{p=k+1}^{\infty} 2^{-p} b_{p}\right)^{-1}
\end{gathered}
$$

$$
A=\sum_{p=0}^{\infty} 2^{-p} b_{p}
$$

Equation (15) is used to eliminate $s$ in (14) to obtain the free energy as a function of temperature.

The internal energy per spin is

$$
U=-\frac{\partial}{\partial \beta} 2^{-N} \ln Q_{N}(\mu, \beta)
$$


When the change of variable indicated above is made the operator $\frac{\partial}{\partial \beta}$ becomes $\frac{\partial}{\partial \beta}+\frac{\partial s}{\partial \beta} \frac{\partial}{\partial s}$, since $s$ depends explicitly upon $\beta$. Thus

$$
\begin{aligned}
U & =-\left(\frac{\partial}{\partial \beta}-\beta^{-1}(s+1) \frac{\partial}{\partial s}\right) 2^{-N} \ln Q_{N} \\
& =\frac{1}{4} \beta^{-1} \sum_{k=0}^{\infty} 2^{-k}-\beta^{-1}(s+1) \sum_{k=0}^{\infty} 2^{-k}\left(s+A^{-1} \sum_{p=k+1}^{\infty} 2^{-p} b_{p}\right)^{-1},
\end{aligned}
$$

which is, by (15)

$$
U=\frac{1}{2} \beta^{-1}-(s+1) A .
$$

$-A$ is the internal energy per spin in the ground state so we calculate the internal energy from this value

$$
\varepsilon=U+A=\frac{1}{2} \beta^{-1}-s A .
$$

The magnetic properties of the spherical hierarchical model follow from the Hamiltonian in an external magnetic field $H$, which is taken to be

$$
\mathscr{H}_{N}=H_{N}+\mu_{0} H \sum_{j=1}^{2^{N}} \mu_{j}
$$

where $\mu_{0}$ is the magnetic moment of an individual spin.

We note that this may be written in terms of the normal coordinates as

$$
\mathscr{H}_{N}=H_{N}+\mu_{0} H 2^{N} X_{1} .
$$

Which is the same as before except for the linear $X_{1}$ dependence. The integral in the partition function is also easily performed and yields

$$
\mathscr{Q}_{N}(\beta, \mu, H)=Q_{N}(\mu, \beta) \exp \left(\frac{1}{4} 2^{N} \beta^{2} \mu_{0}^{2} H^{2}\left(\mu-\beta h_{N}\right)^{-1}\right) .
$$

The free energy per spin is

$$
-2^{-N} \beta \psi_{N}=\ln \mathscr{Q}_{N}(\mu, \beta, H)=\ln Q_{N}(\mu, \beta)+\frac{1}{4} \beta^{2} \mu_{0}^{2} H^{2}\left(\mu-\beta h_{N}\right)^{-1}
$$

where $\mu$ is determined as a function of $\beta$ for fixed $H$ by

$$
1=-\frac{\partial}{\partial \mu} \ln \mathscr{Q}_{N}(\beta, \mu, H) \text {. }
$$


The Magnetization $M$ is

$$
M=\beta^{-1} \frac{\partial}{\partial H} \ln \mathscr{Q}=\frac{1}{2} \mu_{0}^{2} H A^{-1}\left(s+A^{-1} \sum_{N+1}^{\infty} 2^{-p} b_{p}\right)^{-1} .
$$

The Magnetic Susceptibility is

$$
\chi=\frac{\partial M}{\partial H}=\frac{1}{2} \mu_{0}^{2} A^{-1}\left(s+A^{-1} \sum_{N+1}^{\infty} 2^{-p} b_{p}\right)^{-1} .
$$

\section{Analysis of a Particular Example}

Dyson's study of the hierarchical model centered upon an analysis of a particular choice of the $b$ 's. With the choice

$$
b_{p}=2^{(2-\alpha) p}, \quad p \neq 0, \quad b_{0}=0,
$$

one expects that the behavior of this model will correspond to an Ising model with an interaction strength of $n^{-\alpha}$ for $n^{\text {th }}$ neighbor spins. We put this choice into the Eqs. (14) and (15) and obtain:

and

$$
\begin{aligned}
A=\sum_{p=1}^{\infty} 2^{-p} 2^{(2-\alpha) p} & =2\left(2^{\alpha}-2\right)^{-1} \\
A^{-1} \sum_{p=k+1}^{\infty} 2^{-p} 2^{(2-\alpha) p} & =2^{(1-\alpha) k}
\end{aligned}
$$

$$
\begin{aligned}
2^{-N} \beta \psi_{N} & =\frac{1}{4} \sum_{k=0}^{N} 2^{-k} \ln \beta A\left(s+2^{(1-\alpha) k}\right), \\
\beta A & =\frac{1}{4} \sum_{k=0}^{N} 2^{-k}\left(s+2^{(1-\alpha) k}\right)^{-1} .
\end{aligned}
$$

It would appear that there is no difficulty in taking the infinite limit. One simply sets $N=\infty$ in the expressions (23) and (24). This procedure, however, assumes that there is always an unambiguous connection between $s$ and $\beta$.

For any value of $N$ the function

$$
g_{N}(s)=\frac{1}{4} \sum_{k=0}^{N} 2^{-k}\left(s+2^{(1-\alpha) k}\right)^{-1}
$$

takes on all positive values and is monotone decreasing when its argument ranges from $s=-2^{(1-\alpha) N}$ to $s=\infty$. If $g_{N}(0)$ is finite the values of $\beta$ between $g_{N}(0) / A$ and infinity are all crowded into a small region $-2^{(1-\alpha) N}<s<0$. In the limit of infinite $N$ this region shrinks to zero and 
thus, if $g \infty(0)$ is not infinite, we have the value $s=0$ corresponding to a range of values of $\beta$.

This is the mathematical manifestation of the phase transition phenomenon. At the point $s=0$, the inverse temperature $\beta$ is not an analytic function of $s$ in the limit of infinite $N$. In the limit $N \rightarrow \infty, s=0$ for all $\beta<\beta_{c}$ where

$$
\beta_{c}=\frac{1}{4} A^{-1} \sum_{k=0}^{\infty} 2^{-(2-\alpha) k}=\frac{1}{2}\left(2^{\alpha}-2\right)\left(4-2^{\alpha}\right)^{-1} .
$$

This defines the critical temperature.

It has been verified by direct computation that this critical temperature lies between the bounds established by Dyson for the true hierarchical model. We note, again consistent with Dyson and Joyce, that the phase transition exists only for $1<\alpha<2$. For $\alpha<1$ the ground state energy per spin diverges and for $\alpha>1$ the ground state energy per spin diverges and for $\alpha>2$ the interaction falls so rapidly in distance that the transition temperature is infinite.

To establish the critical exponents, we wish to analyze the behavior of the spherical hierarchical model in the neighborhood of the critical point. Let us reckon $\beta$ away from the critical $\beta_{c}$. From (24) and (25) we have

$$
\beta_{c}-\beta=\frac{1}{4} S A^{-1} \sum_{p=0}^{\infty} 2^{-(2-\alpha) p}\left(s+2^{(1-\alpha) p}\right)^{-1} .
$$

Expansion of this expression for small $s$ gives

$$
\beta_{c}-\beta \simeq \frac{1}{4} s A^{-1} \sum_{p=0}^{\infty} 2^{-(3-2 \alpha) p}=s\left(2^{\alpha}-2\right)\left(8-4^{\alpha}\right)^{-1}
$$

which converges only if $1<\alpha<3 / 2$.

If $3 / 2<\alpha<2$ we must perform further analysis. To this end let

$$
s=2^{(1-\alpha) u}
$$

and therefore

$$
u=\frac{1}{1-\alpha} \ln _{2} s .
$$

Substituting this into (26) we obtain

$$
\beta_{c}-\beta=\frac{1}{4} A^{-1} \sum_{p=0}^{\infty} 2^{-(2-\alpha) p}\left(1+2^{(1-\alpha)(p-u)}\right)^{-1} .
$$

Now let $u=L+\Delta$ where $L$ is an integer. Substituting this into (27) and changing variables in the sum leads to

$$
\beta_{c}-\beta=\frac{1}{4} A^{-1} 2^{-(2-\alpha)(L+\Delta)}\left[\sum_{p=-L}^{\infty} 2^{-(2-\alpha)(p-\Delta)}\left(1+2^{(1-\alpha)(p-\Delta)}\right)^{-1}\right]
$$


which may be written

$$
\begin{aligned}
\beta_{c}-\beta= & \frac{1}{4} A^{-1} S^{(2-\alpha) /(\alpha-1)}\left[\sum_{p=-\infty}^{\infty} 2^{-(2-\alpha)(p-\Delta)}\left(1+2^{(1-\alpha)(p-\Delta)}\right)^{-1}\right] \\
& -\sum_{-\infty}^{-L-1} 2^{-(2-\alpha)(p-\Delta)}\left(1+2^{(1-\alpha)(p-\Delta)}\right)^{-1} .
\end{aligned}
$$

The second term on the right may be shown to be proportional to $s^{(2 \alpha-3) /(\alpha-1)}$ and thus is negligible in the limit of small $s$ for $3 / 2<\alpha<2$. We therefore examine

$$
G(\Delta)=\sum_{p=-\infty}^{\infty} 2^{-(2-\alpha)(p-\Delta)}\left(1+2^{(1-\alpha)(p-\Delta)}\right)^{-1} .
$$

Note that $G(\Delta)$ has the following properties:

1) $G(\Delta)=G(\Delta+1)$.

2) $G(\Delta+2 \pi i /(\alpha-1) \ln 2)=e^{2 \pi i(2-\alpha) /(\alpha-1)} G(\Delta)$.

According to property 1) we may expand $G$ in a Fourier Series.

where

$$
G(\Delta)=\sum_{n=-\infty}^{\infty} a_{n} \exp 2 i n \pi \Delta,
$$

$$
a_{n}=\int_{-1 / 2}^{1 / 2} G(\Delta) \exp (-2 i n \pi \Delta) d \Delta .
$$

We now consider the contour integral

$$
g_{n}=\int_{c} G(\Delta) \exp (-2 \pi i n \Delta) d \Delta
$$

integrated around the following closed contour in the complex $\Delta$ plane:

I. along the line $\operatorname{Im} \Delta=0$ from $\operatorname{Re} \Delta=-1 / 2$ to $\operatorname{Re} \Delta=1 / 2$.

II. along the line $\operatorname{Re} \Delta=1 / 2$ from $\operatorname{Im} \Delta=0$ to $\operatorname{Im} \Delta=2 \pi / \alpha-1) \ln 2$.

III. along the line $\operatorname{Im} \Delta=2 \pi /(1-\alpha) \ln 2$ from $\operatorname{Re} \Delta=-1 / 2$ to $\operatorname{Re} \Delta=1 / 2$.

IV. along the line $\operatorname{Re} \Delta=-1 / 2$ from $\operatorname{Im} \Delta=2 \pi /(\alpha-1) \ln 2$ to $\operatorname{Im} \Delta=0$.

This closed contour encircles only a single pole of the single term $p=0$ in the $G(\Delta)$ sum. This pole is located at $\Delta=\pi i /(\alpha-1) \ln 2$. By the residue theorem we have

$$
g_{n}=[(2 \pi i /(\alpha-1) \ln 2)][\exp i(2-\alpha) \pi /(\alpha-1)]\left[\exp 2 \mathrm{n} \pi^{2} /(\alpha-1) \ln 2\right] .
$$

Property 1) of $G(\Delta)$ assures that the contributions to the integral (30) along II and IV cancel. By property 2 ) we see that the integral along III 
is a multiple of the integral along I, and of course, the integral along I is just $a_{n}$. Thus

and

$$
a_{n}\left(1-\left[\exp (2 \pi i(2-\alpha) /(\alpha-1)]\left[\exp 4 n \pi^{2} /(\alpha-1) \ln 2\right]\right)=g_{n}\right.
$$

$$
a_{n}=(\pi /(\alpha-1) \ln 2)\left(\sin \left((2-\alpha) \pi /(\alpha-1)-2 i n \pi^{2} /(\alpha-1) \ln 2\right)\right)^{-1} .
$$

We retain only the constant term and the first harmonic in the Fourier series and rewrite in terms of $s$ using

$$
\sin 2 n \pi \Delta=\sin 2 n \pi(L+\Delta)=\sin 2 n \pi(\ln 1 / s) /(\alpha-1)
$$

$G(s)$

$=\pi \frac{\left[1+4 \exp \left(-2 \pi^{2} /(\alpha-1) \ln 2\right) \sin (2-\alpha) \pi /(k-1) \sin \left(2 \pi \ln \frac{1}{s} /(\alpha-1)+(2-\alpha) \pi /(\alpha-1)\right)\right]}{((\alpha-1) \ln 2 \sin (2-\alpha) \pi /(\alpha-1))}$.

Strictly speaking there is no limit as $s \rightarrow 0$. The coefficient of the troublesome term is so small, however, that from an experimental (i.e. a computer simulation) point of view it is undetectable. We shall use only the first term for the remainder of this analysis.

If $\alpha=3 / 2$, we find that the sum in (28) diverges at the lower limit, and asymptotically therefore

$$
\beta_{c}-\beta=\frac{1}{4} A^{-1} s \ln \frac{1}{s}=\frac{1}{2}(\sqrt{2}-1) s \ln \frac{1}{s} .
$$

Thus there are three regions of interest in the expansion of $\beta_{c}-\beta$ as a function of $s$ near $s=0$ :

$$
\begin{aligned}
& \beta_{c}-\beta \simeq s\left(2^{\alpha}-2\right)\left(8-4^{\alpha}\right)^{-1}, \quad 1<\alpha<3 / 2, \\
& \beta_{c}-\beta \simeq 1 / 2(\sqrt{2}-1) s \ln _{2} 1 / s, \quad \alpha=3 / 2 \text {, } \\
& \beta_{c}-\beta \simeq 1 / 8 s^{(2-\alpha) /(\alpha-1)}\left(2^{\alpha}-2\right) a_{0}, \quad 3 / 2<\alpha<2 \text {. }
\end{aligned}
$$

For $\beta_{c}-\beta$ small and positive we have the connection between $s$ and $\beta$ provided by the inverse of these expressions

Where

$$
\begin{array}{ll}
s \simeq\left(\beta_{c}-\beta\right)\left(8-4^{\alpha}\right) /\left(2^{\alpha}-2\right), & 1<\alpha<3 / 2, \\
s \simeq 2\left(\beta_{c}-\beta\right) /(\sqrt{2}-1) \ln _{2}\left(\beta_{c}-\beta\right)^{-1}, & \alpha=3 / 2, \\
s \simeq \Lambda(\alpha)\left(\beta_{c}-\beta\right)^{(\alpha-1) /(2-\alpha)}, & 3 / 2<\alpha<2 .
\end{array}
$$

$$
\Lambda(\alpha)=\left[8 /\left(2^{\alpha}-2\right) a_{0}\right]^{(\alpha-1) /(2-\alpha)} .
$$

From the Eq. (16), we calculate $\varepsilon$, the internal energy per spin as functions of the variables $s$ and $\beta$. 
For all $\alpha, \beta>\beta_{c}$ implies $s=0$ and the specific heat is a constant independent of temperature. The specific heat is

$$
\begin{aligned}
C_{v} & =-k \beta^{2} \frac{d \varepsilon}{d \beta} \\
& =\frac{k}{2}+A k \beta^{2} \frac{d s}{d \beta} .
\end{aligned}
$$

For $\beta_{c}-\beta$ small and greater than zero (just above the critical temperature) we have

$$
\begin{array}{ll}
C_{v}=\frac{k}{2}\left(1-\left(8-4^{\alpha}\right) / 2\left(4-2^{\alpha}\right)^{2}\right), & 1<\alpha<3 / 2, \\
C_{v}=\frac{k}{2}\left(1-\left(\left(4-2^{3 / 2}\right)^{2} \ln \left(\beta_{c}-\beta\right)^{-1}\right)^{-1}\right), & \alpha=3 / 2, \\
C_{v}=\frac{k}{2}\left(1-\left(\beta_{c}-\beta\right)^{(2 \alpha-3) /(2-\alpha)} \Lambda(\alpha)\left(2^{\alpha}-2\right) /\left(4-2^{\alpha}\right)\right), & (3 / 2<\alpha<2) .
\end{array}
$$

To complete the picture on the critical behavior we now examine the spherical hierarchical model in a magnetic field.

We spezialize Eqs. (19) and (20) to the particular case, take the limit of infinite $N$ and obtain:

$$
\begin{gathered}
\ln \mathscr{Q}_{N}(\mu, \beta, H)=\ln Q_{N}(\mu, \beta)+\frac{1}{4} \beta^{2} \mu_{0}^{2} H^{2} A^{-1} s^{-1} \\
1=\frac{1}{4} \beta^{-1} A^{-1} \sum_{k=0}^{\infty} 2^{-\alpha k}\left(s+2^{(1-\alpha) k}\right)^{-1}+\frac{1}{4} \mu_{0}^{2} H^{2} A^{-2} s^{-2}
\end{gathered}
$$

or

$$
\beta=\left[\sum_{k=0}^{\infty} 2^{-\alpha k}\left(s+2^{(1-\alpha) k}\right)^{-1}\right]\left[1-4 A\left(\frac{1}{4} \mu_{0}^{2} H^{2} s^{-2} A^{-2}\right)\right]^{-1} .
$$

The presence of a magnetic field destroys the phase transition because there is a unique connection between $S$ and $\beta$ for any positive value of $\beta$.

The spontaneous magnetization is the value of $M$ as $H \rightarrow 0^{+}$. One sees from (31) that if $s>0$ as $H \rightarrow 0$ the value of $\beta$ is unaffected by the presence of the field. This limit will always lead to a value of $\beta$ which is less than $\beta_{c}$. On the other hand, consider the limit $s \rightarrow 0, H \rightarrow 0, H / s \rightarrow \lambda$. In this limit from (31) we have

$$
\beta=\beta_{c}\left(1-\frac{1}{4} \mu_{0}^{2} A^{-2} \lambda^{2}\right)^{-1}
$$

or,

$$
\lambda=\left(2 A / \mu_{0}\right)\left(1-\beta / \beta_{c}\right)^{1 / 2}
$$


and

$$
M=\frac{1}{2} A^{-1} \mu_{0}^{2} \lambda=\mu_{0}\left(1-\beta / \beta_{c}\right)^{1 / 2}
$$

The spontaneous magnetization is thus the same as the classical CurieWeiss theory.

Above the critical temperature at zero magnetic field the magnetic susceptibility is

$$
\chi=\frac{1}{2} \mu_{0}^{2} A^{-1} S^{-1}
$$

which by the previous analysis is

$$
\begin{array}{ll}
\chi=\mu_{0}^{2}\left(8-4^{\alpha}\right)^{-1}\left(\beta_{c}-\beta\right)^{-1}, & 1<\alpha<3 / 2, \\
\chi=\mu_{0}^{2}\left(\beta_{c}-\beta\right)^{-1} \ln _{2}\left(\beta_{c}-\beta\right)^{-1}, & \alpha=3 / 2, \\
\chi=\mu_{0}^{2}\left(2^{\alpha}-2\right) \Lambda^{-1}(\alpha)\left(\beta_{c}-\beta\right)^{(1-\alpha) /(2-\alpha)}, & 3 / 2<\alpha<2 .
\end{array}
$$

These critical exponents for the specific heat, magnetic susceptibility (both above and below the critical temperature) and spontaneous magnetization are identical with those obtained by Joyce for the longrange spherical Ising model.

\section{Spin Correlations}

We shall conclude our analysis of the properties of the spherical hierarchical model with a calculation of the spin-spin correlation matrix, which is defined to be

$$
\varrho_{i j}=\left\langle\mu_{i} \mu_{j}\right\rangle .
$$

This correlation matrix will have strange properties because the hierarchical model is not invariant to a cyclic relabeling of spin coordinates and thus the correlation matrix will not be a function of $i-j$ alone. We shall calculate the full correlation matrix $P$, which is

$$
P=\left\langle u u^{\dagger}\right\rangle \text {. }
$$

From standard gaussian analysis of variance one sees that this matrix is the inverse of the matrix $\mu I-\beta A_{N}$. This matrix will have the same hierarchical character as $A_{N}$, that is it will satisfy the same recurrence relation as $A_{N}$, but with replacement

$$
\begin{aligned}
& b_{0} \text { replaced by } \mu-\beta b_{0} \\
& b_{n}, \quad n \neq 0 \text { replaced by }-\beta b_{n} .
\end{aligned}
$$

Thus we concentrate on inverting $A_{N}$. 
We may construct the inverse of $A_{N}$ by assuming that the inverse has the same recursive form as $A_{N}$. That is we assume that $A_{N}^{-1}$ is of the form:

$$
A_{N}^{-1}= \begin{cases}A_{N-1}^{-1}+e_{N} C_{N-1} & e_{N} C_{N-1} \\ e_{N} C_{N-1} & A_{N-1}^{-1}+e_{N} C_{N-1}\end{cases}
$$

where $e_{N}$ is to be determined.

The $A_{N}$ 's and $C_{N}$ 's have the following useful properties:

and

$$
\begin{aligned}
C_{N-1}^{2} & =2^{N-1} C_{N} \\
A_{N-1} C_{N-1} & =\left(\sum_{P=0}^{N-1} 2^{-p} b_{p}\right) C_{N-1}
\end{aligned}
$$

$$
\mathrm{C}_{N-1} \mathrm{~A}_{N-1}^{-1}=\left(\sum_{P=0}^{N-1} 2^{-p} b_{p}\right)^{-1} C_{N-1}
$$

because every vector in $C$ is an eigenvector of $A$.

With these properties we multiply $A_{N}$, as given in (10), by the assumed form for $A_{N}^{-1}$. The condition that this matrix invert $A_{N}$ is found to be

$$
\begin{aligned}
e_{N} & =-2^{-2 N} b_{N}\left[\left(\sum_{p=0}^{N} 2^{-p} b_{p}\right)\left(\sum_{k=0}^{N-1} 2^{-k} b_{k}\right)\right]^{-1} \\
& =2^{-N}\left[\left(\sum_{p=0}^{N} 2^{-p} b_{p}\right)^{-1}-\left(\sum_{p=0}^{N-1} 2^{-p} b_{p}\right)^{-1}\right] .
\end{aligned}
$$

Thus we generate the inverse recurrence relation by replacing $b_{N}$ with

$$
2^{N} b_{N}\left[\left(\sum_{p=0}^{N} 2^{-p} b_{p}\right)^{-1}-\left(\sum_{p=0}^{N-1} 2^{-p} b_{p}\right)^{-1}\right]
$$

in the expression (10).

Let us now calculate the first row of the correlation matrix $P$. The first row of the matrix $A_{N}$ is

$$
A_{1,2^{p-1}+1}=A_{1,2^{p-1}+2}=\cdots=A_{1,2^{p}}=\sum_{k=p}^{N} 2^{-2 k} b_{k} \text { for all } \varrho>1
$$

making the above replacement for the $b_{N}$ we find:

$$
\begin{aligned}
P_{1,2^{p}+1} & =P_{1,2^{p}+2}=\cdots=P_{1,2^{p}} \\
& =\frac{1}{2}\left[\sum_{k=p}^{N} 2^{-k}\left[\left(\sum_{n=0}^{k} 2^{-n} b_{n}\right)^{-1}-\left(\sum_{n=0}^{k=1} 2^{-n} b_{n}\right)^{-1}\right] .\right.
\end{aligned}
$$


Replacing the $b$ 's by their counterparts given in (32) we obtain

$$
\begin{aligned}
P_{1,2^{p-1+1}} & =P_{1,2^{p-1}+2}=\cdots=P_{1,2^{p}} \\
& =\frac{1}{2}\left[\sum_{k=p}^{N} 2^{-k}\left(\mu-\beta \sum_{n=0}^{k} 2^{-n} b_{n}\right)^{-1}-\sum_{k=p}^{N} 2^{-k}\left(\mu-\sum_{n=0}^{p-1} 2^{-n} b_{n}\right)^{-1}\right] .
\end{aligned}
$$

In the hierarchical chain a given spin has a fixed correlation with every spin which is in a different half of the chain, a fixed correlation with every spin which is in a different quarter of the chain, etc. The correlation coefficient given above may be interpreted as the first row of the correlation matrix or as the correlation coefficient of a pair of spins which are in the same $2^{p}$ fraction, but different $2^{p+1}$ fractions, of the chain.

In the particular case, with the usual change of variable, the expression above reads:

$$
\begin{aligned}
P_{1,2^{p-1}+1} & =\cdots=P_{1,2^{p}} \\
& =1-\frac{1}{4} \beta^{-1} A^{-1}\left[\sum_{k=0}^{p} 2^{-k}\left(s+2^{(1-\alpha) k}\right)^{-1}+2^{-p}\left(s+2^{(1-\alpha) p}\right)\right] .
\end{aligned}
$$

We can draw a rough correspondence between this spin correlation and one from a translation invariant Hamiltonian. The expression (33) represents the correlation between a pair of spins which are in the same block of $2^{p}$ spins, but are not in the same block of $2^{p+1}$ spins. If the chain sites are equally spaced the distance from a given spin site to the end of the block of $2^{p}$ spins which it occupies will be roughly.

$$
\begin{aligned}
& n \sim 2^{p-1} \\
& p \sim \ln _{2} 2 n .
\end{aligned}
$$

If we compute the elements of the correlation matrix (32) at the critical temperature $\left(\beta=\beta_{c}, s=0\right)$ we find

$$
P_{1,2^{p-1}+1}=\cdots=P_{1,2^{p}}=\frac{1}{4} 2^{-(2-\alpha) p}\left[2^{2 \alpha}-4\right] .
$$

Using the above rough correspondence between $P$ and distance we find

$$
P(n) \sim(2 n)^{-(2-\alpha)} .
$$

Thus the spin-spin correlation goes roughly as a power law at the transition temperature. The power is $-(2-\alpha)$, which is again consistent with Joyce. 


\section{Summary}

The spherical version of the hierarchical model has a phase transition which satisfies the inequalities established by Dyson for the true hierarchical model. Dyson was able to connect the hierarchical model to the long range Ising model using the Griffiths inequalities. A particular case of the spherical hierarchical model may be directly compared to the spherical long range Ising model since both are exactly soluble. These models are strikingly similar. Both models depend on a single parameter which governs the rate of decrease of the interaction between spins. As a function of this parameter all critical exponents are calculated to be the same, and the range of the parameter over which a phase transition exists is the same. This perhaps indicates that the similarity between the two models is deeper than Dyson expected.

The critical behaviors of the infinite spherical hierarchical model is governed by the connection between $\beta$ and $s$. In general this connection is given by (15) and is

where

$$
\beta A=\frac{1}{4} \sum_{k=0}^{\infty} 2^{-k}\left(S+A^{-1} \sum_{p=k+1}^{\infty} 2^{-p} b_{p}\right)^{-1}
$$

$$
A=\sum_{p=0}^{\infty} 2^{-p} b_{p} .
$$

The ground state energy of the spherical hierarchical model is $A$ (per spin). This sum must converge, and it is this requirement in the special case which requires that $\alpha>1$. A phase transition occurs if the right hand side is upper bounded for all positive values of $s$. The maximum value of the right hand side is

$$
\beta_{c} A=\frac{1}{4} \sum_{k=0}^{\infty} 2^{-k}\left(A^{-1} \sum_{p=k+1}^{\infty} 2^{-p} b_{p}\right)^{-1} .
$$

In order that there be a phase transition this sum must converge.

If we consider a hierarchy of $2^{L}$ finite hierarchical systems each containing $2^{N}$ spins, the ground state energy is

$$
E_{0}=-2^{N+L} \sum_{p=0}^{N+L} 2^{-p} b_{p}
$$

The ground state energy per system of $2^{N}$ spins is

$$
2^{-L} E_{0}=-2^{N} \sum_{p=0}^{N+L} 2^{-p} b_{p}
$$


If we subtract this from the ground state self energy of an individual $2^{N}$ spin system we obtain (per spin)

$$
\left(-2^{N} \sum_{p=0}^{N} 2^{-p} b_{p}-E_{0} 2^{-L}\right)=2^{N} \sum_{p=N+1}^{N+L} 2^{-p} b_{p} .
$$

In the limit $L \rightarrow \infty$ this is the sum which governs the existence of a phase transition.

Thus, for the hierarchical model to make sense it must have a finite ground state energy per spin. For it to have a phase transition the interaction energy (per system) of an infinite system of systems of finite size must diverge as the number of spins becomes infinite.

A number of other special cases where

$$
b_{p}=2^{(2-\alpha) p} \mathrm{p}^{m}
$$

have been examined, but will not be reported in detail. We do, however, wish to point out that all cases examined have exactly the same critical behavior as the case analyzed here. No attempt has been made to define the limits, but it seems safe to say that critical exponents are "universal" over a very wide class of $b$ 's.

In all of the cases analyzed the critical exponents are very weak functions of temperature, as in the special case. It is tempting to conjecture that this abnormal critical behavior is connected with the lack of translation invariance in the hierarchical Hamiltonian.

Acknowledgement. The author wishes to acknowledge valuable discussions with C. J. Thompson and $\mathrm{K}$. W. Thompson during the course of this work.

\section{References}

1. Dyson, F. J.: Commun. math. Phys. 12, 91 (1969).

2. Griffiths, R. B.: J. Math. Phys. 8, 418 (1967).

3. Berlin, T.H., Kac, M.: Phys. Rev. 86, 821 (1952)

J. B. McGuire

Department of Physics

Florida Atlantic University

Boca Raton, Florida 33432, USA 